\title{
Multi-Channel Fusion Model of Perception System in Lower Extremity Exoskeleton Suits and it's Implementation
}

\author{
Xianggang Zhang, Caoyuan Zhao \\ School of Aeronautics and Astronautics, University of \\ Electronic Science and Technology of China \\ Chengdu, Sichuan province, China \\ csxgzhang@uestc.edu.cn
}

\begin{abstract}
-the human exoskeleton suit is a complex servo system characterized by high degree of human-machine coupling, which means that exoskeleton suit would be able to follow or synchronize with the human body movement. The perception system, which is responsible for sensing the wearer's movement and movement intention, plays a key role in realizing human-machine coupling. How to build a perception system in human exoskeleton suit? In the paper, a multi-channel perception model is described, through which the data from multi-channels is gathered, fused, and then wearer's movement and movement intention are sensed and transmitted to the controller. In order to easily gather the information from various sensors and devices, interface management layer is introduced. Meanwhile, it also can provide the support for redundancy, fault tolerance, and adaptation in dynamic environments. After being gathered, how do the data from heterogeneous sensors be integrated? How would the data been interpreted into accurate and comprehensive movement posture? In the paper, the three methods, which are time correlation, posture perception and movement prediction, are used to implement the data fusion. Meanwhile, a prototype based on this model was implemented in our human exoskeleton suit. In the paper, the layout of sensors, hardware, software, algorithms, and test of the prototype are explained.
\end{abstract}

Keywords-Exoskeleton; Human-machine coupling; Multichannel

\section{INTRODUCTION}

The term of exoskeleton originally comes from the biology, which refers to the external skeleton that supports and protects an animal's body. The human exoskeleton suit is an automatic electro-mechanical system, which is worn to augment the wear's strength and endurance, and to extend the wear's capabilities. Human exoskeleton suit is a complex integrated combination of mechanics, computer technology, control technology, micro-drive technology, materials technology and other disciplines.

Various research topics about human exoskeleton suit attract the researchers' attention all over the world, including Kanagawa Institute of Technology (KIT) , University of Tsukuba (Tsukuba,), UC Berkeley, Massachusetts Institute of Technology( MIT), Nanyang Technological University, and so on. The applications of human exoskeleton suit could be divided into two mainly aspects: firstly, the exoskeleton technology is used to enhance the functions of the body, for

\author{
Qingxia Lin, Qifang Zhou \\ School of Aeronautics and Astronautics, University of \\ Electronic Science and Technology of China \\ Chengdu, Sichuan province, China \\ 915607142@qq.com
}

example, BLEEX[1] and RoboKnee[2]; secondly, the exoskeleton technology is adopted to make up for the defects or weakness of the human body functions, mainly used in healthcare and rehabilitation, for example, LOKOMAT [3]and ReWalk[4].

Compared to the other robots, human exoskeleton suit is characterized by tightly human-machine coupling. It means that the suit would have capability to follow or synchronize the wearer's movement. So human-machine coupling is the essential character of human exoskeleton suit.

The basis and premise to realize man-machine coupling is that the machine should be able to accurately know the wearer's movement posture and movement intention. So the perception system plays a key role in human exoskeleton suit. The role of perception system is as the interface between exoskeleton suit and human, as shown in Fig.1. Its functions could be summarized into 4 points, i.e. acquisition of information, transmission of data, transfer of format, and comprehension of semantics. According to the level of manmachine coupling, the data flow can be divided into one-way or two-way. If system just needs the machine to follow human body, only one-way data flow is needed; if mutual cooperation and coordination between human and machine are needed, the two-way data flow is needed.

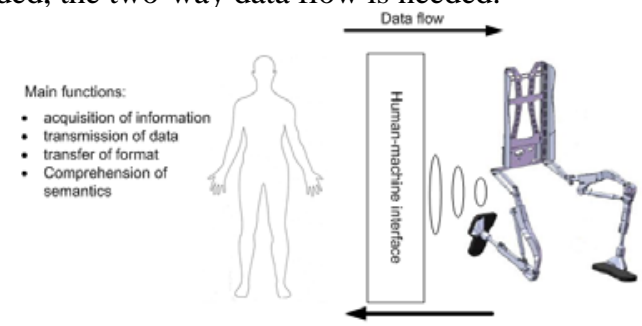

Figure 1. human-machine interface

This article is intended to establish a multi-channel intelligent perception system through multi-channel input, data fusion and prediction, then to realize information complementary, conflicts elimination, and accurate perception of the wearer's movement. In the paper, firstly, we present a model of perception system, which mainly contains interface management layer and data fusion layer. The purpose of interface management layer is to build an opening architecture. The purpose of data fusion layer is to integrate data and perceive the wearer's movement. Then, the implementation of a prototype is explained. 
In the rest of the paper, section 2 is related to the related works. In section 3, the multi-channel fusion model is explained. In section 4 , the details of a perception system in lower extremity exoskeleton is explained. At last, we summarize the paper.

\section{RELATED WORKS}

The perception system of human exoskeleton suit mainly includes various sensors distributed all over the body and movement recognition technologies. In Berkeley lower extremity exoskeleton (BLEEX) of UC Berkeley, 8 encoder and 16 linear acceleration sensors are adopted to determine the angle, angular velocity, angular acceleration, while foot switches, load distribution sensors and single-axis force sensors are used to gather the relevant information. A physical network, named as ExoNET, was designed to enable the central controller to interact with distributed sensors, and provide high-speed real-time communication [1, 5, 6]. In HAL from University of Tsukuba of Japan, The rotary encoders are used to measure the angle of knee and hip. The EMG sensors are used to estimate the muscle activity and torques of joint. The foot pressure sensor is used to collect the floor reaction force [7, 8, 9]. In East China University of Science and Technology, the researchers have developed a reliable and durable sensing boots of exoskeleton suit to accurately measure gait through placed pressure sensors in the appropriate location of the bottom of boots [10].

In Chinese Academy of Sciences, a multi-sensors perception system was designed to obtain the human lower limb movement intention. The system includes force sensor, the ground reaction force sensor, and the motor encoder [11]. In order to improve the dynamic response frequency of the lower limb, the system also proposed a novel signal line prediction algorithm based on time series analysis [12]. The other related researches and universities include the Massachusetts Institute of Technology, U.S. SARCOS, Zhejiang University, China Science and Technology University [9].

In robot systems, the perception system mainly includes the visual, auditory, tactile, and other devices. The robot's visual system is used to identify objects, which includes optical sensing system, ultrasonic sensing system, and etc. The robot hearing is the voice recognition system. The robot tactile system is composed by the tactile sensor and the control section, which can detect the object that robots contact with. Pressure sensors can be detected pressure between the robot and the object $[13,14]$.

Body Area Network (BSN) is a local area network to collect information of body, which is worn on the body or implanted in the human body. The study included the production of physiological sensors, the building of the body area network, data fusion, context awareness, and etc. $[15,16]$

\section{MUlTi-ChanNel FUSION MODEL}

Multi-channel fusion model, shown in the Fig.2, includes interface management layer (IM layer) and data fusion layer (DF layer). After the physical signal of sensors is acquired and preprocessed, the signals are transmitted to interface management layer, which provides common accessing interface and separate virtual devices from physical devices. Next step is data fusion layer, which gathers the data, calculate, deduces the wearer's posture, and then transfers the information to the controller. The controller controls the actuators to assist wearer's movement.

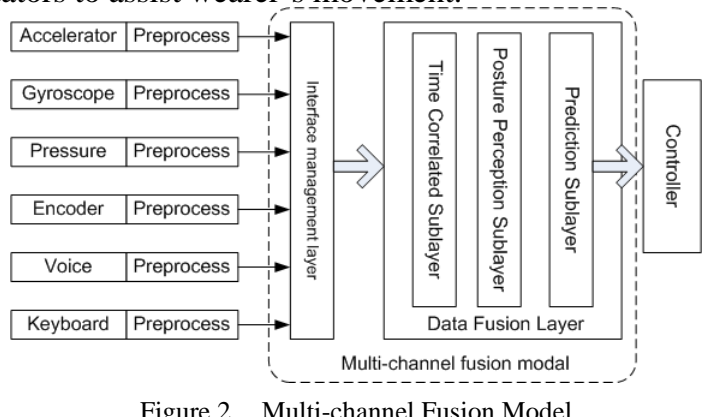

The IM layer mainly provides 2 functions. One is devices management. The other is to provide isolation function between physical devices and DM layer.

The function of device management provides the registration and management for all sensors and input devices. For example, when a new sensor is installed into the system, it would register its profile to IM layer, including device driver, parameters and so on. Meanwhile, the functions, such as driver management, calibration table management and so on, are also provided.

On the other hand, the IM layer is an isolation layer, which separates the DF layer from the details of physical devices. It provides standard querying interface to access physical devices for DF layer. The perception system can use the standard interfaces to query data from a sensor, regardless of the details of the sensor. It also separates the virtual devices from physical devices. One virtual device can be mapped to different physical device. Through the adaptive mapping, IM layer can provides redundancy, fault tolerance and adaption for dynamic environments. By the following scenario (Fig.3), we can understand this function. For example, if some sensor go wrong or disappear or being updated, the layer can switch to another device while the application remains silence. The only thing needed to rewrite the mapping table. We can take a scene. In the exoskeleton suit, there is an accelerometer and gyroscope sensor for measuring the speed and posture of body, there is also a GPS receiver for location service. If accelerometer is wrong, GPS receiver can be used to measure the speed of movement, and if there is no signal of GPS, accelerator and gyroscope can be used to locate.

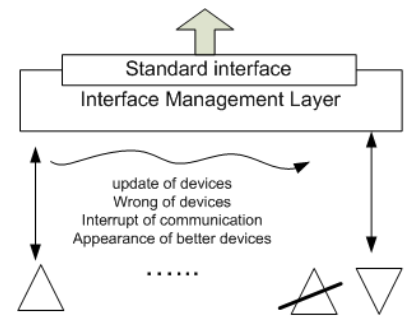

Figure 3. scenario of adaptive mapping 
In perception system, the sensors and input devices are heterogeneous; data format are various; and semantics of data are different. How to integrate the data from the heterogeneous devices, and how to extract the useful information from various data flows? This is a key problem which must be addressed, namely the multi-channel integration. It is the responsibility of DF layer.

Multi-channel integration need to address the following questions, or provide the following functions. (1) data from one sensor is only about a part of body, which is needed to integrate with others into the whole posture of a human body; (2) information complement each other and correction of information; (3) prediction of movement intention and movement trend.

In the model, there are three steps in data fusion layer. They are time correlated sub-layer, posture perception sublayer and movement prediction sub-layer. In time correlated sub-layer, some data are grouped together to represent the state of the body in some time point according to the time tag; In posture perception sub-layer, the posture parameters of body are derived from the data of various sensors. In this sub-layer, the laws of human movement, kinematics, dynamics, and physiological mechanics will be used to deduce wearer's movement state. In prediction layer, according to the laws of human movement, expert database, historical information, and the current parameters of movement, the position and orientation in the next perception period will be predicted. Through the data fusion module, the perception system will show an accurate and comprehensive wearer's movement state to the controller.

\section{Prototype Based on The Model}

\section{A. Layout of Sensors and Input devices}

All the sensors and input devices in system include pressure sensors, gyroscopes, encoders, pressure switches, keyboard, and microphone. The foot pressure sensors and pressure switches are installed in forefoot and heels of each shoe for gait recognition. A rotary incremental encoder is installed on each knee joint for measuring knee angle. The gyroscopes are mounted on the shank and the thigh to measure the angular velocity. Meanwhile, there are a keyboard and a microphone, which can gather the wearer's instructions. The function and layout of the sensors and input devices are shown in table I.

TABLE I. THE FUNCTION AND LAYOUT OF THE SENSORS

\begin{tabular}{|l|c|c|}
\hline \multicolumn{1}{|c|}{ Device Name } & Function & Layout \\
\hline Microphone, keyboard & Instruction input & shoulder \\
\hline gyroscope & Angular velocity measurement & $\begin{array}{c}\text { Shank \& } \\
\text { thigh }\end{array}$ \\
\hline encoder & Angle measurement & Knee joint \\
\hline Pressure switch & Pressure measurement & $\begin{array}{c}\text { Sole of shoe, } \\
\text { heel of shoe }\end{array}$ \\
\hline Pressure sensors & Pressure measurement & $\begin{array}{c}\text { Sole of shoe, } \\
\text { heel of shoe }\end{array}$ \\
\hline
\end{tabular}

\section{Design of Software}

Layered architecture is adopted in Software, as shown in Fig.6. From bottom, first one is sensor layer. Second is preprocessing layer, which amplified and interpreted original signals. The next is interface management layer, which separate upper application from physical sensor and manage all sensors in the system. The functions of this layer are explained as section 3 . The main data structures in this layer are various tables, such as mapping table, register table and calibration table. On the top is data fusion layer, which recognize and predict body movement, then provide them to control module. This layer contains three sub-layers, which are time correlated sub-layer, posture perception sub-layer and predict sub-layer. There is a data queue pool between interface management layer and data fusion layer.

The architecture of interface management layer is as shown in Fig.7. From bottom up, the first modules are driver management and communication, which are responsible for managing the drivers of all the devices and communication. 
The second is devices mapping module. Through update a mapping table, it directs each query to available sensors. Through devices mapping module, applications can use different device to finish the same function in spite of changes of the environment. The top is interface module, which mainly provides query interface and register interface. The query interface is used to get information, and the register interface is used to register a new device. All the modules are under the management of controller.

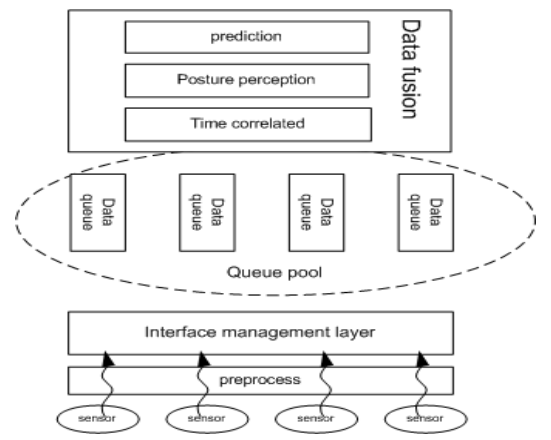

Figure 6. Architecture of Software

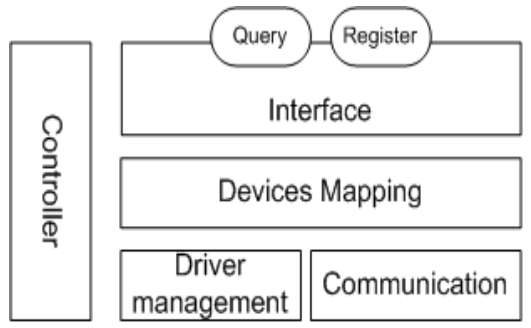

Figure 7. Architecture of IM layer

\section{Algorithms in DF Layer}

\section{1) Time Correlated Sub-Layer}

Since the difference of response time and processing speed of each channel, the structure of each input event or data have a field to record the time, named timestamp. The integration mechanism will use the timestamp to associate the input data with each other. In the system, in each period of perception, all the latest data are grouped based on their timestamp. Furthermore, based on the control algorithms, the data on various time points can be gathered into a group, not just the latest data.

\section{2) Posture Perception Sub-Layer}

Because the normal walking is slow and low frequency, recognition model of body posture is established as Fig.8. The recognition of body posture includes 3 steps. First step shown as solid arrow is recognition of whole body state, such as standing, low-speed walking, high-speed walking, and going upstairs and so on. Second step shown as dash arrow is recognition of gait, such as left leg supporting \& right leg swing. The third step shown as triangle is recognition of movement parameters, such as the angular velocity and angle of knee. The Fig. 8 only shows a state transition from standing state. Each movement state has a state transition diagram.

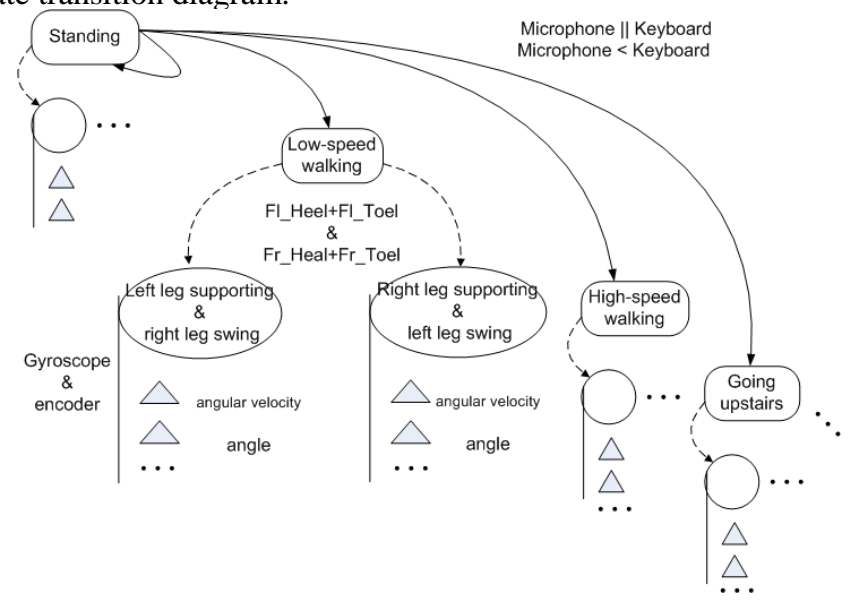

Figure 8. A state transition diagram in posture perception sub-layer

a) Recognition of the whole body state

In first step, the exoskeleton suit recognizes the whole body state based on the instructions from microphone and keyboard. Before the wearer changes his whole body state, for example from stopping to walking, the instruction from microphone or keyboard will be issued to CCM. Furthermore, we define that keyboard has higher Priority than microphone's.

\section{b) Gait Recognition}

In the step of gait recognition, which phase of the posture can be recognized. For example, we can use the foot pressure sensors to recognize the gait.

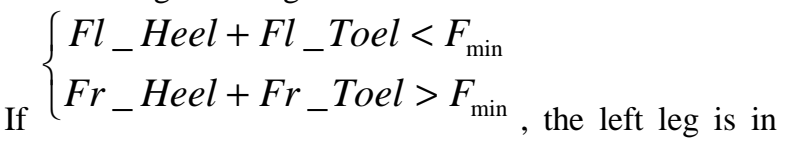
swing phase and the right leg is in stance phase.

If, $\left\{\begin{array}{l}F l_{-} \text {Heel }+F l_{-} \text {Toel }>F_{\text {min }} \\ F r_{-} \text {Heel }+F r_{-} \text {Toel }<F_{\text {min }}, \text { the right leg is in }\end{array}\right.$ swing phase and the left leg is in stance phase.

The Fl_Heel, Fl_Toel, Fr_Heel, Fr_ Toel respectively represent the outputting value of pressure sensor in heel of left shoe, in the frontfoot of left shoe, in heel of right shoe, and in the frontfoot of right shoe. Fmin is a threshold of the outputting value of pressure sensors. During different whole body state, there are different phases of gait.

Because of the rapid change of Pressure sensor signals and the constraint of the shoe structure, a low-pass filter is designed to decrease the misjudgment of gait. The filter using a first order inertia link, the algorithm is expressed as follows, $G_{f l t}=\frac{1}{1+T_{f l t} S}$, in which, $T_{f l t}=0.025 s$, it is a time constant of filter.

\section{c) Parameter Recognition}


In the step of parameter recognition, the detailed movement parameters can be recognized. We attached the gyroscopes on the thigh and shank, and installed encoder on the knee joint. Using these sensors, we can measure the angle and angular velocity of knee joint.

The encoder on the knee joint is an absolute encoder. The output voltage is range from $0.5 \mathrm{~V}$ to $4.5 \mathrm{~V}$, which is corresponds to the rotation cycle of 360 degree.

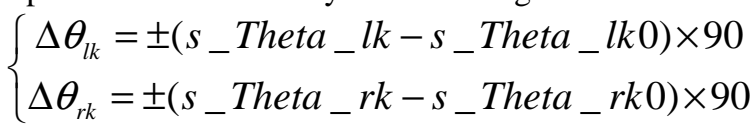

$\Delta \theta_{l k}, \Delta \theta_{r k}$ are Angle increment of left knee joint and right knee joint.

$s_{-}$Theta_lk, $s_{-}$Theta_rk are the original signal measured by encodes on left knee joint and right knee joint.

$s_{-}$Theta_lk0,s_Theta_rk0

measured by encodes on left knee joint and rig

Furthermore, based on the data from encoders and gyroscopes, the angular velocity of the knee joint can be calculated.

\section{d) Parameters based Machining Learning}

A person's movement is characterized by arbitrary. Machine-learning technology can be adopted to get characters, which is customized for different user and different situation. In our system, machining learning is used to determine the threshold value of gait analysis. Because the gait only be divided into swing phase and stance phase, specified number of clusters is 2, and then experiment EM cluster algorithm on it, the result is shown in Fig.9.

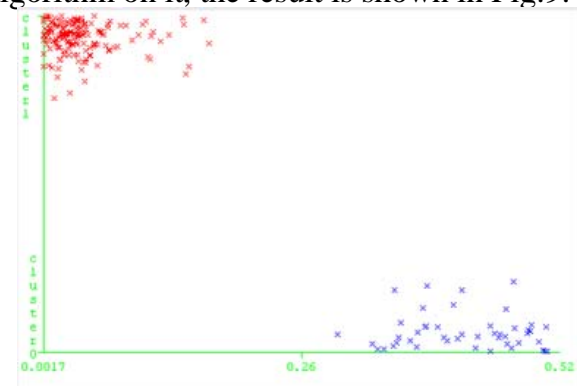

Figure 9. Cluster result of 2-phase without class

After clustering analysis, the correspondence relationship of sample data and clustering can be obtained. One is named as Lst Rsw, the other is named as Lsw Rst. The result is as table II. The $\mathrm{fl}$ attribute denotes the addition of $F l_{-} H_{e e l}+F l_{-}$Toel , the fr attribute denotes the addition of $\mathrm{Fr} \_$Heel $+\mathrm{Fr}_{-}$Toel , L_encode and $\mathrm{R}$ _encode respectively represent the outputs from encoders in left knee and right knee. Lst_Rsw represents that left leg is in stance phase and right leg is in swing phase. Lsw_Rst represents that left leg is in swing phase and right leg is in stance phase. Then the data in table II are utilized to train the classifier.
TABLE II. PART OF 2-Phase GAIT DATA SAMPLE

\begin{tabular}{|c|c|c|c|c|}
\hline Fl & fr & L_encode & R_encode & Class \\
\hline 0.4461 & 0.0495 & -0.0439 & 47.2026 & Lst_Rsw \\
\hline 0.4419 & 0.0933 & -0.283 & 40.42 & Lst_Rsw \\
\hline 0.4364 & 0.1161 & -0.214 & 38.4692 & Lst_Rsw \\
\hline 0.1486 & 0.8128 & 1.3062 & 42.9592 & Lsw_Rst \\
\hline 0.1271 & 0.8401 & 1.0681 & 43.2633 & Lsw_Rst \\
\hline
\end{tabular}

The decision tree for the C4.5 algorithm is illustrated in Fig.10. This result is very simple, based on the total pressure of left heel and left frontfoot, the phase of gait can be correctly judged (the judgment of right leg is similar). So machine learning algorithms have a good effect to refine the identification of the leg movements. Furthermore, the algorithm also can filter some amount of redundant sensing data rather than the employer to choose.

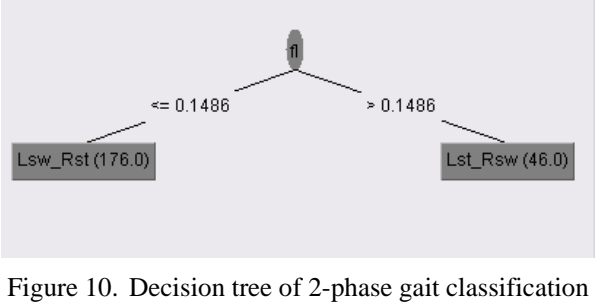

\section{3) Prediction Layer}

In prediction layer, intelligent forecasting techniques in perception system are used to improve the real-time and speed of response of the control system, to reduce constraints on the human body, and to improve man-machine coupling. In the prediction layer, three methods are used to achieve intelligent prediction. They are the prediction method based on the kinematics and dynamics, prediction methods based on expert database and prediction methods based on machine learning.

Prediction method, based on the kinematics and dynamics, is a set of corresponding equations of movement and mechanical equations based on kinematics, dynamics and physiology. The body movement has some certain regularity and all parts of the body are correlated each other in movement. The expert system can be used to reveal their relationship and law through statistical data. Expert system can provide an important reference for posture prediction. Prediction methods based on machine learning can be used to get prediction customized for each wearer and various situations. A human movement is characterized by arbitrary. It will be different on different time and place. Beside of formulas derived from kinematics and dynamics, as well as the law from statistics, machine-learning can be adopted to get characters, which is customized for different user and different situation.

\section{TEST}

After wearing the exoskeleton suit, the wearer remains stationary standing firstly, then walks, then stops for a moment, then walk again. The data from sensors and input 
devices are gathered, analyzed, and then deduced the wearer's movement.

The instruction from microphone or keyboard will be used to judge the whole body state. Before the wearer begins to stop or walk, he will issue the instruction from keyboard or microphone.

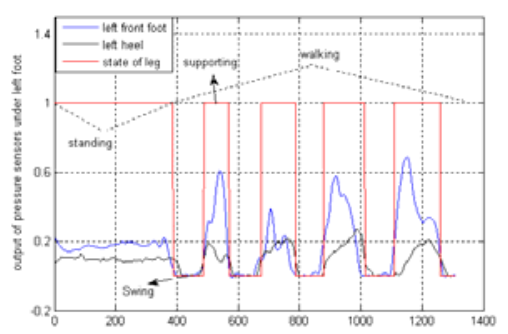

Figure 11. pressure sensors of left foot

After the whole body state is judged, gait recognition can be processed. For example, the output of left foot pressure is shown in Fig.11. According to the formula

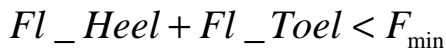
or $F l_{-}$Heel $+F l_{-}$Toel $>F_{\text {min }}$, the supporting state and swing state can be judged in the walking process.

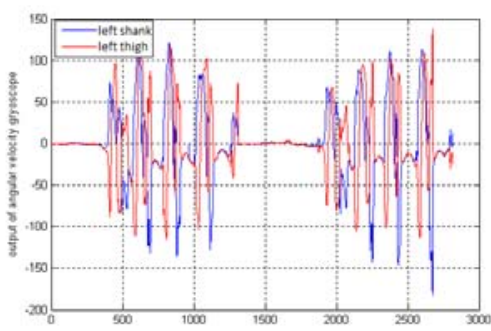

Figure 12. gyroscopes of left leg

Fig.12 shows the output data of gyroscopes attached on thigh and shank. Combined with the angle from encoder, the angular velocity and angle of knee joint can be calculated. Then we can obtain detailed parameters of body posture.

\section{CONCLUSION}

The human exoskeleton suit is characterized by high degree human-machine coupling. The perception system plays a key role as the interface between human and exoskeleton suit. How to build the perception system? Firstly, the perception system would be ease to integrate various devices including sensors, keyboard and so on. Secondly, the perception system would be able to interpret various data into wearer's movement and movement intention. So, in the model, IM layer and DF layer are proposed. Furthermore, what are the algorithms in DF layer? It will be depend on the control method. In the paper, the algorithms in the prototype are explained. At last, the prototype is test to verify the reasonability.

\section{REFERENCES}

[1] Steger R, Sung Hoon Kim , Kazerooni H. Control scheme and networked control architecture for the Berkeley lower extremity exoskeleton (BLEEX). Proceedings of the 2006 IEEE International Conference on Robotics and Automation. P3469 - 3476. May 1519,2006

[2] Jerry E. Pratt, Benjamin T. Krupp, Christopher J. Morse. The RoboKnee: An Exoskeleton for Enhancing Strength and Endurance During Walking. Proceeding of the 2004 IEEE International Conference on Robotics \& Automation. P.2430-2435 Vol.3, 26 April1 May 2004.

[3] The homepage of Hocoma. available: http://www.hocoma.com/.

[4] The homepage of Argo Medical Technologies Ltd. available: http://www.argomedtec. Com.

[5] Aaron M. Dollar,HughHerr.Lower Extremity Exoskeletons and Active Orthoses:Challenges and State-of-the-Art. IEEE Transactions on Robotics, Vol. 24, No. 1, Feb. 2011.

[6] H. Kazerooni,R. Steger. The Berkeley Lower Extremity Exoskeleton.Journal of Dynamic Systems, Measurement, and Control.March 2006, Vol. 128 /25

[7] Kawamoto H., SankaiY..Comfortable Power Assist Control Method for Walking Aid by HAL-3.2002 IEEE SMC TP1B2.

[8] Kawamoto, H., Sankai, Y., "Power Assist System HAL-3 for gait Disorder Person”, ICCHP, July 2002, Austria.

[9] Robert Bogue. Exoskeletons and robotic prosthetics: a review of recent developments. Industrial Robot: An International Journal 36/5 (2009) 421-427.

[10] Cao Heng, He Cheng-Kun, Meng Xian-Wei et al. Structure optimization analysis for exoskeleton foot[J]. Journal of Engineering Design, 2010, 17(1): 35-39.

[11] Sun Jian, Yu Yong, Ge Yunjian, Chen Feng, Shen Huan-hua. Research on multi-sensors perceptual system of wearable power assist leg based article in a journal.

[12] Sun Zhao-jun, Yu Yong, Ge Yun-jian. Study on Sensing Information Forecasting of Wearable Power Assist Walking Legs Based on Time Series Analysis. Journal of Transduction Technology. Vol.22(3), March 2009

[13] Tong Libiao, $\mathrm{Xu}$, Kejun, Mei Tao. A survey of multi-sensor integration and fusion techniques of robotics gripper. Journal of Hefei University of Technology. Vol.24(1). Feb. 2001.

[14] Sascha Kolski, Mobile Robots: Perception \& Navigation, ISBN 386611-283-1.

[15] Ji-Bing Gong;Rui Wang);Li Cui. Research Advances and Challenges of Body Sensor Network. Journal of Computer Research and Development. Vol.47(5) May 2010.

[16] Mark A. Hanson, Harry C. Powell Jr., Adam T. Barth, Kyle Ringgenberg, Benton H. Calhoun, James H. Aylor, and John Lach, Body Area Sensor Networks: Challenges and Opportunities. Computer, 42, Issue:1P.58-65. 